Ophthalmologica

\title{
Dietary Tocopherol Supplementation after Trabeculectomy and Phacotrabeculectomy: Double-Blind Randomized Placebo-Controlled Trial
}

\author{
David Goldblum a, b Alexander Meyenberg ${ }^{\mathrm{a}} \quad$ Daniel Mojon ${ }^{\mathrm{c}}$ \\ Christoph Tappeiner $^{\mathrm{a}}$ Beatrice E. Frueh ${ }^{\mathrm{a}}$ \\ Departments of Ophthalmology, a University of Bern, Inselspital, Bern, and ${ }^{b}$ University Hospital Basel, \\ University of Basel, Basel, and 'Department of Strabismus and Neuro-Ophthalmology, Kantonsspital St. Gallen, \\ St. Gallen, Switzerland
}

\section{Key Words}

Vitamin E - Glaucoma - Surgery · Trabeculectomy •

Wound-healing $\cdot$ Failure $\cdot$ Complications

\begin{abstract}
Purpose: The vitamin E compound $\alpha$-tocopherol inhibits fibroblast growth in vitro. To evaluate its potential benefit in preventing failure of glaucoma filtration surgery, we prospectively investigated the outcome of filtering surgery with postoperative dietary $\alpha$-tocopherol supplementation. $\mathbf{P a}$ tients and Methods: Thirty-nine patients (39 eyes) with primary open-angle glaucoma or pseudoexfoliative glaucoma were randomly assigned to two post-(phaco)-trabeculectomy treatment groups. Daily oral intake of $300 \mathrm{mg} \alpha$-tocopheryl-acetate (absorbed as $\alpha$-tocopherol in the intestine) for 2 months was compared to a placebo preparation in a double-blind trial. The follow-up time was 1 year. Success was defined as intraocular pressure (IOP) $\leq 18 \mathrm{~mm} \mathrm{Hg}$ with no medication, no needling revision, and no subconjunctival injection. Results: In the tocopherol group, the relative risk for trabeculectomy failure decreased from 1.58 at 2 weeks $(p=0.95)$ to 1.0 at 1 year. Success rates $(67-90 \%)$ and
\end{abstract}

IOP reduction (mean 39 to 53\%) were statistically comparable in the two groups. Considering postoperative complications, no relevant differences were found. Discussion: Trabeculectomy and phacotrabeculectomy outcome did not show any differences between $\alpha$-tocopherol-supplemented patients and the placebo group.

Copyright $\odot 2009$ S. Karger AG, Basel

\section{Introduction}

Filtering and nonfiltering procedures are still the main glaucoma surgeries of choice today. Despite their success, there is a steady rate of failure owing to progressive scarring in the subconjunctival tissues. To overcome this problem, wound-healing-modifying agents are sometimes applied during surgery. Nevertheless, potential sight-threatening complications limit the generous use of mitomycin $\mathrm{C}$ in glaucoma procedures.

In the search for antiscarring alternatives, Larrosa et al. [1, 2], Pinilla et al. [3, 4] and our group [5, 6] focused on compounds of the vitamin E family. Of these, $\alpha$-tocopherol, $\alpha$-tocopheryl acetate, $\alpha$-tocopheryl succinate,

\section{KARGER}

Fax +4161306 1234 E-Mail karger@karger.ch www.karger.com (c) 2009 S. Karger AG, Basel

0030-3755/09/2234-0228\$26.00/0

Accessible online at:

www.karger.com/oph
David Goldblum, MD

Department of Ophthalmology, University Hospital Basel

University of Basel

$\mathrm{CH}-4031$ Basel (Switzerland)

Tel. +41 61265 2525, Fax +41 61265 8745, E-Mail dgoldblum@uhbs.ch 
and $\alpha$-tocotrienol exhibited antiproliferative and antifibrotic effects in vitro and in a rabbit model of glaucoma filtration surgery [1-6].

In this study, we report the results of our double-blind prospective study, where we hoped to modify the postoperative outcome of trabeculectomy or phacotrabeculectomy with $\alpha$-tocopherol dietary supplementation.

\section{Patients and Methods}

Inclusion criteria of consecutive patients were medically uncontrolled primary open-angle or pseudoexfoliative glaucoma necessitating filtering surgery - with or without lens implantation. Progressive visual field changes associated with glaucoma and open anterior chamber angles in gonioscopy had to been proven.

Exclusion criteria were legal blindness in the fellow eye, previous ocular surgery in either eye, laser trabeculoplasty within the last 6 months, neovascular glaucoma, drug addiction, known psychiatric or intestinal diseases, fat malabsorption disease, vitamin E supplementation 3 months before surgery, and unsafe contraception in young women. A detailed history, best corrected visual acuity, slit-lamp biomicroscopy with optic nerve head evaluation and Goldmann applanation tonometry were recorded in all study participants the day before surgery.

All patients were recruited in the Department of Ophthalmology, Inselspital, University of Bern, and gave their written informed consent before inclusion in the study. The study was approved by the local ethical committee and conformed to the provisions of the Declaration of Helsinki.

One surgeon performed all trabeculectomies as follows: in all cases, a fornix-based opening of the conjunctiva and Tenon's capsule, and a limbus-based $4 \times 4-\mathrm{mm}$ scleral flap was prepared reaching the clear cornea. A sclerotomy containing the cornea and trabecular meshwork was done using a motor trephine (1.5 $\mathrm{mm}$ in diameter). A peripheral iridectomy was always performed. After suturing of the scleral flap with two nylon 10-0 sutures, the conjunctiva was closed with two polyglactin 8-0 sutures and a Ushaped nylon 10-0 suture at the 12 o'clock position. No mitomycin $\mathrm{C}$ or 5-fluorouracil was used during the surgical procedure. Postoperative standard topical therapy included atropin (1\%) ointment for the first week and antibiotic-steroid ointment and drops (dexamethasone, neomycin and polymyxin B) initially 6 times daily which were then tapered off over 5 weeks.

If a cataract with a visual acuity of less than 0.8 was present, a combined surgical procedure - phacotrabeculectomy - was offered. After preparation of the scleral flap, a corneal tunnel starting at the flap base was created followed by routine phacoemulsification (phaco-chop) with posterior chamber lens implantation. Sclerotomy, peripheral iridectomy, wound closure and postoperative medical therapy were then performed as described above.

Study participants were randomly assigned in a double-blind fashion to either the tocopherol or the control/placebo group. Subjects in the tocopherol group were treated with one $300-\mathrm{mg}$ capsule of all-rac- $\alpha$-tocopheryl acetate $\left(\right.$ Ephynal ${ }^{\circledR}$, Bayer, Switzerland) daily for 2 months. Patients in the control group received identical-looking placebo capsules. Follow-up examinations were

Tocopherol Supplementation after

Trabeculectomy scheduled at 2 weeks, 2 months and 1 year postoperatively. Intraocular pressure (IOP), filtering bleb appearance, postoperative complications and interventions were recorded.

Successful trabeculectomy or phacotrabeculectomy was defined as intraocular pressure (IOP) $\leq 18 \mathrm{~mm} \mathrm{Hg}$ with no antiglaucomatous medication, no needling revision and no subconjunctival injection. Success rates, IOP levels, and postoperative complications were compared between the tocopherol group and the control group 2 weeks, 2 months and 1 year postoperatively using Fisher's exact test and the Mann-Whitney U test. The relative risk of trabeculectomy and phacotrabeculectomy failure was calculated. Differences with a first-order error of $\mathrm{p}<0.05$ were considered statistically significant.

\section{Results}

A total of 41 patients (41 eyes) were included into the study: 19 participants were randomly assigned to the tocopherol group, 22 to the control group. Two subjects had to be excluded on the first postoperative day before initiation of treatment due to anterior chamber bleeding (76-year-old female with pseudoexfoliative glaucoma; 68 -year-old male with primary open angle glaucoma). Both patients received a tissue plasminogen activator injection.

The demographic and clinical characteristics did not differ significantly between the 19 subjects of the tocopherol group and the remaining 20 subjects of the control group (table 1). However, patients in the tocopherol group had more frequently pseudoexfoliative glaucoma and tended to suffer from more advanced glaucoma (larger/ deeper visual field defects, higher preoperative IOP, more antiglaucomatous drugs preoperatively). On the other hand, patients in the control group had been on presurgical antiglaucomatous therapy for a longer period of time and had received a combined surgical procedure (phacotrabeculectomy) slightly more often.

All study participants were repeatedly re-examined for at least 2 months after trabeculectomy or phacotrabeculectomy at the Department of Ophthalmology, University of Bern. One-year follow-up was available for $18 / 19$ subjects of the tocopherol group and $18 / 20$ subjects of the control group.

Trabeculectomy and phacotrabeculectomy failures were as follows for the tocopherol group versus the control group: 3 versus 3 eyes at 2 weeks; 3 versus 2 eyes at 2 months; 0 versus 1 eye at 1 year postoperatively. In the tocopherol group, 4 filtering blebs were encapsulated (and subsequently needled or/and injected with mitomycin C) and 2 blebs were inconspicuous, whereas in the control group 4 blebs were encapsulated (and subsequent- 
Table 1. Demographic and clinical characteristics of the $\alpha$-tocopherylacetate and placebo-treated groups

\begin{tabular}{|c|c|c|c|}
\hline & Tocopherol $(\mathrm{n}=19)$ & Control $(\mathrm{n}=20)$ & p value ${ }^{1}$ \\
\hline \multicolumn{4}{|l|}{ Demographic characteristics } \\
\hline Female/male & $9 / 10$ & $8 / 12$ & 0.751 \\
\hline Age, years & $74.0 \pm 9.1$ & $69.1 \pm 11.7$ & 0.121 \\
\hline \multicolumn{4}{|l|}{ Clinical characteristics } \\
\hline POAG/PXG & $6 / 13$ & $11 / 9$ & 0.200 \\
\hline BCVA (decimal); mean and range & $0.64 ; 0.05-1.0$ & $0.66 ; 0.03-1.2$ & 0.910 \\
\hline Refraction, SE, dptr & $-0.91 \pm 3.62$ & $-1.03 \pm 3.15$ & 0.683 \\
\hline Mean defect Octopus VF, dB & $17.03 \pm 8.03$ & $11.49 \pm 8.73$ & 0.065 \\
\hline Loss variance Octopus VF, $\mathrm{dB}^{2}$ & $38.17 \pm 30.29$ & $25.19 \pm 19.77$ & 0.220 \\
\hline Duration of antiglaucomatous therapy before surgery, years & $4.9 \pm 6.6$ & $7.1 \pm 5.6$ & 0.094 \\
\hline Preoperative number of antiglaucomatous drugs (maximum $=6$ ) & $2.6 \pm 1.1$ & $2.2 \pm 1.2$ & 0.175 \\
\hline Preoperative IOP, $\mathrm{mm} \mathrm{Hg}$ & $25.6 \pm 9.5$ & $22.9 \pm 4.7$ & 0.619 \\
\hline Trabeculectomy/combined trabeculectomy and lens implantation & $14 / 5$ & $12 / 8$ & 0.501 \\
\hline Previous laser trabeculoplasty & 4 & 7 & 0.480 \\
\hline
\end{tabular}

POAG = Primary open angle glaucoma; PXG = pseudoexfoliative glaucoma; BCVA = best corrected visual acuity calculated by $\log$ MAR (log of the minimum angle of resolution) conversion; $\mathrm{SE}=$ spherical equivalent; Octopus VF = visual field in automated perimetry (Octopus 101).

${ }^{1}$ Two-tailed $\mathrm{p}$ by the Mann-Whitney U test and Fisher's exact test.

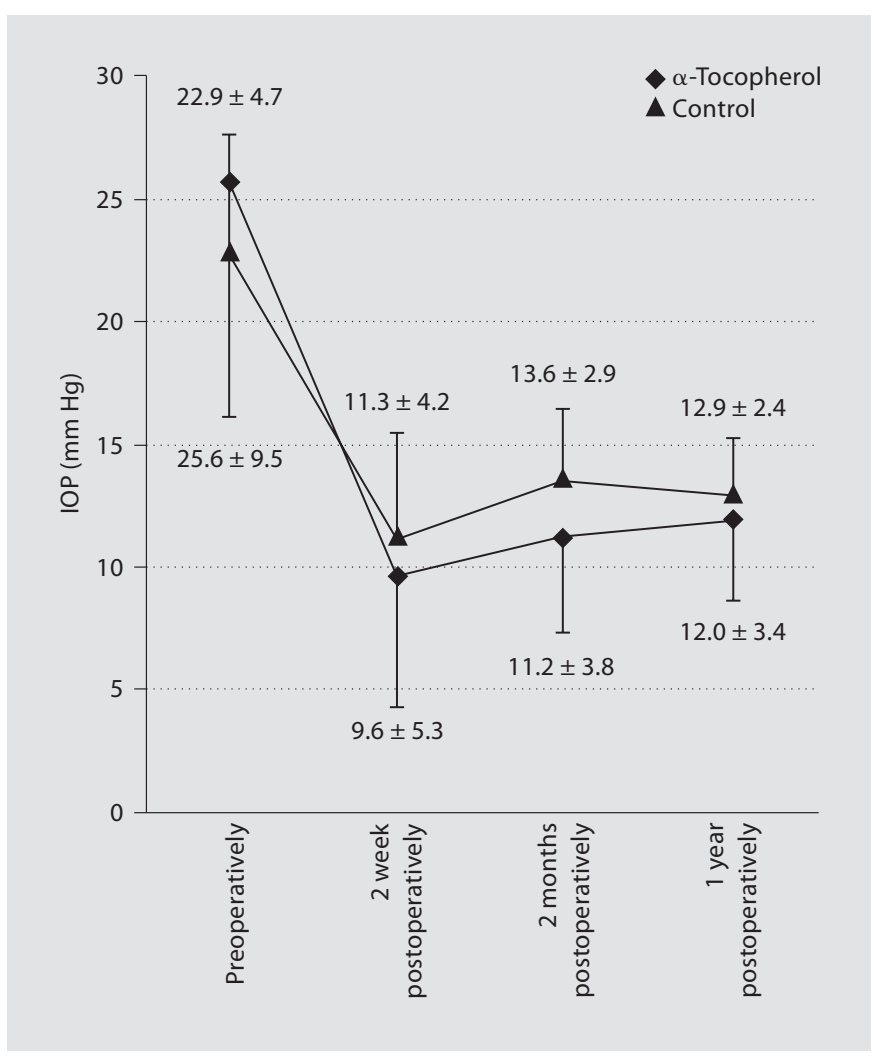

Fig. 1. Graph illustrating the pre- and postoperative IOPs in the $\alpha$-tocopheryl-acetate- and placebo-treated groups (mean IOP \pm $\mathrm{SD}, \mathrm{mm} \mathrm{Hg}$ ). ly needled and/or injected with mitomycin C), 1 flat and 1 inconspicuous. In these 6 unsuccessful cases, the IOP ranged from 19 to $28 \mathrm{~mm} \mathrm{Hg}$ in the tocopherol group and from 19 to $23 \mathrm{~mm} \mathrm{Hg}$ in the control group. The relative risk ratio for trabeculectomy and phacotrabeculectomy failure decreased in the tocopherol group from 1.58 at 2 weeks [ $95 \%$ confidence interval $(\mathrm{CI})=0.30-8.43$ ] to 1.26 at 2 months $(95 \% \mathrm{CI}=0.46-3.46)$, and finally to 1.0 at 1 year postoperatively $(95 \% \mathrm{CI}=0.40-2.52)$.

The success rates of trabeculectomy and phacotrabeculectomy did not differ significantly between the two study groups (tocopherol vs. control): 84 versus $85 \%(\mathrm{n}=$ $19 / 20)$ at 2 weeks, 68 versus $75 \%(n=19 / 20)$ at 2 months, and 67 versus $67 \%(\mathrm{n}=18 / 18)$ at 1 year postoperatively (Fisher's exact test, two-tailed $\mathrm{p}=0.731-1.0$ ). Figure 1 presents the preoperative and postoperative IOP levels in successful trabeculectomy and phacotrabeculectomy cases in the two study groups (fig. 1). On average, subjects in the tocopherol group had higher preoperative/pretreatment IOP and lower postoperative IOP levels compared with the control group. At 2 weeks postoperatively, successful trabeculectomy and phacotrabeculectomy cases showed a decrease in IOP of $53.2 \pm 18.1 \%$ (mean \pm $\mathrm{SD}$ ) in the tocopherol group versus $43.8 \pm 17.6 \%$ in the control group (Mann-Whitney $\mathrm{U}$ test, two-tailed $\mathrm{p}=$ 0.186 ). The decrease in IOP was $49.9 \pm 21.3 \%$ (tocopherol) versus $39.1 \pm 20.5 \%$ (control) at 2 months postop- 
Table 2. Distribution of early postoperative complications in the $\alpha$-tocopheryl-acetate- and placebo-treated groups

\begin{tabular}{|c|c|c|c|}
\hline & $\begin{array}{l}\alpha \text {-To- } \\
\text { copherol } \\
(n=19)\end{array}$ & $\begin{array}{l}\text { Control } \\
(\mathrm{n}=20)\end{array}$ & $\begin{array}{l}\mathrm{p} \\
\text { value }^{1}\end{array}$ \\
\hline Wound leak (positive Seidel test) & 3 & 3 & 1.000 \\
\hline Hypotony $(\leq 5 \mathrm{~mm} \mathrm{Hg})$ at week 2 & 3 & 3 & 1.000 \\
\hline Peripheral choroidal detachment & 2 & 0 & 0.231 \\
\hline Flat anterior chamber & 1 & 0 & 0.487 \\
\hline $\begin{array}{l}\text { Total number of eyes with } \\
\text { complications }\end{array}$ & $7(37 \%)$ & $4(20 \%)$ & 0.301 \\
\hline
\end{tabular}

More than one complication can be found in the same eye.

${ }^{1}$ Fisher's exact test (two-tailed $\mathrm{p}$ ) and $\chi^{2}$ test.

eratively $(\mathrm{p}=0.200)$, and $47.3 \pm 19.6 \%$ versus $44.9 \pm$ $13.6 \%$ at 1 year postoperatively $(\mathrm{p}=0.670)$.

A total of 7 eyes in the tocopherol group and 4 eyes in the control group were affected by postoperative complications (Fisher's exact test for tocopherol vs. control, twotailed $\mathrm{p}=0.301)$ : 1 eye had prolonged hypotony alone in both groups, i.e. IOP $\leq 5 \mathrm{~mm} \mathrm{Hg}$ for at least 2 weeks; 1 eye in each group showed wound leak alone, i.e. Seidel test was positive; 2 eyes in each group had wound leak and prolonged hypotony requiring resuturing ( $p>0.999$ for all comparisons). In the tocopherol group, but not in the control group, 2 eyes showed transient peripheral choroidal detachment $(\mathrm{p}=0.231)$ and 1 eye had a flat anterior chamber $(\mathrm{p}=0.487)($ table 2$)$.

\section{Discussion}

Failure of glaucoma surgery still confronts the clinician with a dilemma. In most cases, medical therapy is reinstituted. A significant proportion of these patients are refractory to medical or laser therapy and further surgery is the only option. To prevent this failure, different antifibrotic substances have been tested or are used intraand postoperatively with a varying degree of success [7]. So far, adequate evidence for the prevention of bleb failure has only been shown for mitomycin $C[8,9]$. However, because of its long-term local impairment of ocular healing and defense, mitomycin $\mathrm{C}$ can have serious sightthreatening side effects. A study of the follow-up and complications of perioperative mitomycin C demonstrated that $10 \%$ had late bleb leak, $10 \%$ scleral necrosis, and $5 \%$ hypotonous maculopathy [10]. The incidence of endophthalmitis after antimetabolite-treated trabeculectomy is reported to be $1.1 \%$ for superior trabeculectomies and $8 \%$ for inferior trabeculectomies $[11,12]$.

In an attempt at enlarging the therapeutic armamentarium with a less toxic substance, we tested the hypothesis of bleb failure prevention by adding $300 \mathrm{mg} /$ day of systemic $\alpha$-tocopheryl acetate in a double-blind study. Although the systemic route is unconventional in glaucoma treatment, we opted for this approach.

Fuller et al. [13] showed that the combination of systemic antifibrotic drugs (prednisolone, colchicine and flufenamic acid or diclofenac) was able to prevent fibrosis and bleb failure without significant local or systemic side effects in patients with signs of threatened early bleb failure.

In nonsupplemented humans, plasma $\alpha$-tocopherol concentrations commonly vary between 20 and 35 $\mu \mathrm{mol} / \mathrm{l}$. A daily dosage of $300 \mathrm{mg} \alpha$-tocopheryl acetate (absorbed in the intestine as $\alpha$ ) was chosen since 2-3 times increased plasma levels have been observed in similarly supplemented individuals and these concentrations were shown to be antiproliferative in fibroblast cultures $[5,14]$.

Phacotrabeculectomy and trabeculectomy outcome did not differ between $\alpha$-tocopherol-supplemented individuals and the placebo group. Therefore, daily oral intake of up to $300 \mathrm{mg} \alpha$-tocopherol ( $\alpha$-tocopheryl acetate) seems unlikely to have a relevant beneficial antiscarring effect in glaucoma-filtering procedures. Subconjunctival injection might at best be an alternative application method to be taken into consideration for future studies.

\section{Acknowledgement}

This study was supported by an unrestricted grant from the Swiss National Science Foundation.

References

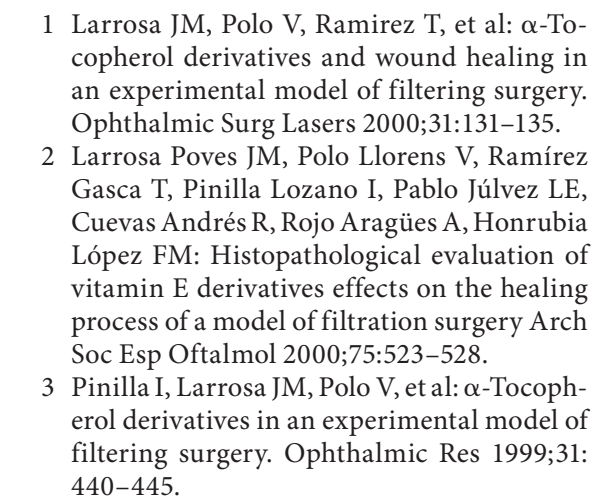

Ophthalmologica 2009;223:228-232 
4 Pinilla I, Piazuelo E, Jimenez P, et al: Inhibitory effect of $\alpha$-tocopherol succinate on fibroblast wound healing. Arch Soc Esp Oftalmol 2000;75:383-388.

$\checkmark 5$ Haas AL, Boscoboinik D, Mojon DS, et al: Vitamin E inhibits proliferation of human Tenon's capsule fibroblasts in vitro. Ophthalmic Res 1996;28:171-175.

6 Meyenberg A, Goldblum D, Zingg JM, et al: Tocotrienol inhibits proliferation of human Tenon's fibroblasts in vitro: a comparative study with vitamin $\mathrm{E}$ forms and mitomycin C. Graefes Arch Clin Exp Ophthalmol 2005; 243:1263-1271.

$\checkmark 7$ Chang MR, Cheng Q, Lee DA: Basic science and clinical aspects of wound healing in glaucoma filtering surgery. J Ocul Pharmacol Ther 1998;14:75-95.
8 Wilkins M, Indar A, Wormald R: Intra-operative mitomycin $\mathrm{C}$ for glaucoma surgery. Cochrane Database Syst Rev 2005; 4: CD002897.

9 Wormald R, Wilkins MR, Bunce C: Postoperative 5-fluorouracil for glaucoma surgery. Cochrane Database Syst Rev 2001;3: CD001132.

10 Singh J, O’Brien C, Chawla HB: Success rate and complications of intraoperative $0.2 \mathrm{mg} /$ $\mathrm{ml}$ mitomycin $\mathrm{C}$ in trabeculectomy surgery. Eye 1995;9:460-466.

11 Higginbotham EJ, Stevens RK, Musch DC, et al: Bleb-related endophthalmitis after trabeculectomy with mitomycin C. Ophthalmology 1996;103:650-656.
12 DeBry PW, Perkins TW, Heatley G, et al: Incidence of late-onset bleb-related complications following trabeculectomy with mitomycin. Arch Ophthalmol 2002;120:297300.

13 Fuller JR, Bevin TH, Molteno AC, et al: Antiinflammatory fibrosis suppression in threatened trabeculectomy bleb failure produces good long-term control of intraocular pressure without risk of sight threatening complications. Br J Ophthalmol 2002;86:13521354.

4 Mickle DA, Weisel RD, Burton GW, et al: Effect of orally administered $\alpha$-tocopherol acetate on human myocardial $\alpha$-tocopherol levels. Cardiovasc Drugs Ther 1991;5:309312 . 